\title{
CÉMENTATION DU NIOBIUM PAR DES SILICIURES DE TITANE ET DE CHROME EN VUE DE RÉALISER DES REVÊTEMENTS PROTECTEURS
}

\author{
J.-M. ALBRECHT, J. AUBRY et B. ROQUES \\ Laboratoire de Métallurgie et Chimie du Solide, associé au C. N. R. S., \\ Faculté des Sciences, 1, rue Grandville, 54, Nancy
}

\begin{abstract}
Résumé. - Les auteurs présentent les premiers résultats d'une étude sur la protection du niobium par des revêtements à base de siliciures. Différents types de revêtements ont été réalisés par cémentation activée. L'un d'entre eux, modifié par le chrome, paraît être intéressant ; il est obtenu en utilisant l'alliage $40 \mathrm{Cr}-60 \mathrm{Si}$ at. \% comme cément et l'iode comme agent activant.

Abstract. - This work is concerned with the development of titanium — and chromium modified silicide coatings suitable for oxidation protection of niobium.

One of these coatings, with chromium as modifier, has exhibited encouraging oxidation behaviour in preliminary tests. It is applied by a pack cementation traitment in a $40 \mathrm{Cr}-60 \mathrm{Si}$ at. \% prealloyed powder with iodine as activator.
\end{abstract}

Cette étude a pour objet la réalisation par cémentation gazeuse de revêtements de siliciures, susceptibles de protéger le niobium et ses alliages contre l'oxydation.

Le disiliciure de niobium pur n'offre pas une protection suffisante et il est nécessaire de le remplacer, au moins en partie, par des siliciures de métaux différents. Parmi ceux-ci, le titane et le chrome ont été particulièrement recommandés [1-4] et nous les avons retenus en priorité.

Le problème était donc de réaliser le plus simplement possible des revêtements associant le silicium à l'un ou à l'autre de ces métaux.

I. Conditions opératoires. - La cémentation activée a été adoptée pour cette étude car elle a déjà manifesté dans de nombreuses applications deux qualités essentielles pour la protection; elle fournit en effet des revêtements qui sont le plus souvent uniformes et bien adhérents au substrat.

$\mathrm{Ce}$ mode opératoire consiste en un traitement thermique dans un cément constitué du (ou des) élément (s) à déposer et d'un réactif, appelé agent activant, qui assure le dépôt par une réaction chimique de transport.

Pour la réalisation des revêtements de siliciures modifiés, un traitement unique représentait la meilleure solution et il était naturel d'examiner d'abord les résultats de la cémentation par des alliages de silicium et de titane ou de silicium et de chrome. Mais pour que ces traitements aient quelques chances de succès, il fallait évidemment que l'agent activant utilisé soit capable d'assurer le transport simultané des deux constituants de ces alliages.

Le choix de ce réactif a été fait en utilisant une méthode indirecte. On sait que les processus thermo- chimiques mis en œuvre dans les cémentations activées reposent sur des réactions réversibles qui sont déclenchées par l'abaissement de l'activité thermodynamique du donneur lors de sa combinaison avec le métal de base ; de ce fait, elles sont liées à la diffusion à l'état solide dans le revêtement. L'équilibre du système solide-gaz formé à partir du cément peut être aussi déplacé sous l'effet d'un gradient de température.

Il est donc possible de vérifier l'efficacité d'un agent activant indépendamment du métal traité, en réalisant des essais de transport du donneur entre deux zones maintenues à des températures différentes.

Cette méthode a montré que tous les siliciures de titane et de chrome peuvent être transportés par réaction avec l'iode et l'influence de la pression de cet halogène et de la composition de l'alliage donneur a été également précisée [5]; le tétrachlorure de silicium convient aussi pour les siliciures de titane mais beaucoup moins bien pour les composés du chrome.

Nous avons donc essayé l'iode et le tétrachlorure de silicium pour les cémentations du niobium par les alliages du titane alors que seul l'iode a été utilisé pour le chrome.

Les traitements ont toujours été effectués dans des enceintes étanches et isothermes, sous une pression partielle contrôlée de l'agent activant. Avant l'introduction de ce réactif et leur scellement, les boîtes de cémentation étaient d'ailleurs soigneusement dégazées.

II. Revêtements modifiés par le titane. - 1 . CÉMENTATION PAR DES ALliages TITANE-SILICIUM. - Le niobium a été traité par une série d'alliages dont les compositions couvraient toute l'étendue du système binaire entre le titane et le silicium purs.

Ces essais ont été réalisés à $1000^{\circ} \mathrm{C}$, avec l'iode et 
le tétrachlorure de silicium comme agents activants. Leurs résultats sont les suivants :

$\begin{array}{ccccc}\text { Donneurs } & \mathrm{Ti} & \mathrm{Ti}_{5} \mathrm{Si}_{3} & \mathrm{TiSi} & \mathrm{TiSi}_{2} \quad \mathrm{Si} \\ \text { Revêtements } & \begin{array}{c}\text { Solution } \\ \text { solide } \\ \text { Nb-Ti }\end{array} & \begin{array}{c}\text { Siliciures de niobium pra- } \\ \text { tiquement } \\ \text { titane }\end{array} & \text { exempts de } \\ & \text { Nb- } & & \end{array}$

Le titane et le silicium ne se déposent jamais simultanément et l'on est amené à conclure que la réalisation de revêtements associant ces deux éléments est impossible en un seul traitement isotherme.

Lorsque la composition du cément se situe dans le domaine $\mathrm{Ti}^{-} \mathrm{Ti}_{5} \mathrm{Si}_{3}$, il se produit uniquement un dépôt de titane, le siliciure se comportant alors comme un diluant inerte.

$\mathrm{Au}$ contraire, tous les donneurs plus riches en silicium donnent lieu essentiellement à une siliciuration du métal de base même si la présence du titane est quelquefois décelable dans une zone très étroite à la surface du revêtement.

Ces deux domaines sont limités par le siliciure $\mathrm{Ti}_{5} \mathrm{Si}_{3}$ qui présente un certain domaine d'homogénéité et dont le comportement dépend ainsi de sa composition. Saturé en titane, il est pratiquement inerte vis-à-vis du niobium, alors que plus riche en silicium, il devient susceptible d'assurer une siliciuration. Lorsque l'iode est utilisé comme agent activant, cette siliciuration est perturbée par une attaque parasite du niobium.

2. DÉPOTS SUCCESSIFS DE TITANE ET DE SILICIUM. Les traitements directs par des alliages titane-silicium ayant échoué, la solution plus classique de dépôts successifs du titane et du silicium a été envisagée. Les deux étapes de ce procédé ont été examinées et d'assez nombreuses difficultés ont été rencontrées dans l'une et l'autre.

Dépôt de titane. - Le traitement du niobium par le titane en présence d'iode est en principe bien adapté à la réalisation d'une zone superficielle de solutions solides; l'iode est un agent de transport efficace et l'interdiffusion des deux métaux est rapide à des températures modérées, voisines de $900 \mathrm{ou} 1000^{\circ} \mathrm{C}$.

Le contrôle de la pureté et de la composition des revêtements est tout de même délicat surtout lorsque l'obtention de solutions solides riches en titane est recherchée et que ce métal est donc utilisé à l'état libre comme donneur.

Le choix de la qualité de ce dernier a alors une grande influence sur la pureté des solutions solides formées. Celles-ci présentent en effet l'inconvénient de dissoudre facilement des éléments comme l'oxygène ou l'azote qui les fragilisent, et il est essentiel d'éviter cette contamination. A cet effet, il est prudent d'exclure le titane divisé à l'état de poudre ou d'éponge et d'utiliser un métal pur sous forme de copeaux; il convient également de purifier soigneusement l'atmosphère de cémentation.

Ces précautions étant prises, il subsiste des diffi- cultés dans le contrôle de la composition et de l'uniformité du revêtement. La diffusion à l'état solide étant très rapide, la composition et l'épaisseur de la zone de solutions solides sont fortement influencées par la vitesse d'apport du donneur à la surface du métal de base; or celle-ci dépend de plusieurs facteurs opératoires.

Les essais effectués dans les mêmes conditions de temps, température et pression d'iode, montrent que le gain de poids et la teneur superficielle en titane des échantillons varient dans le même sens que la concentration du métal dans le donneur. Ainsi l'utilisation d'un cément assez dense de copeaux de petites dimensions permet de réaliser des revêtements épais et pauvres en niobium, alors que la dilution des copeaux dans une poudre inerte, constituée par exemple du siliciure $\mathrm{Ti}_{5} \mathrm{Si}_{3}$, se traduit par un appauvrissement de la zone de diffusion en titane.

D'autre part, dans des essais effectués à $1000^{\circ} \mathrm{C}$ avec un cément non dilué, nous avons constaté que la composition des revêtements variait suivant la pression d'iode, tant que celle-ci restait inférieure à 0,5 atmosphère. C'est seulement au-dessus de cette valeur que le gain de poids des échantillons dépend uniquement de la durée du traitement et croît en fonction de celle-ci suivant une loi parabolique; dans la majeure partie du revêtement, le gradient de concentration en titane en fonction de la pénétration est alors fixé, avec un maximum à la surface compris entre 85 et $90 \%$ en masse (Fig. 1).

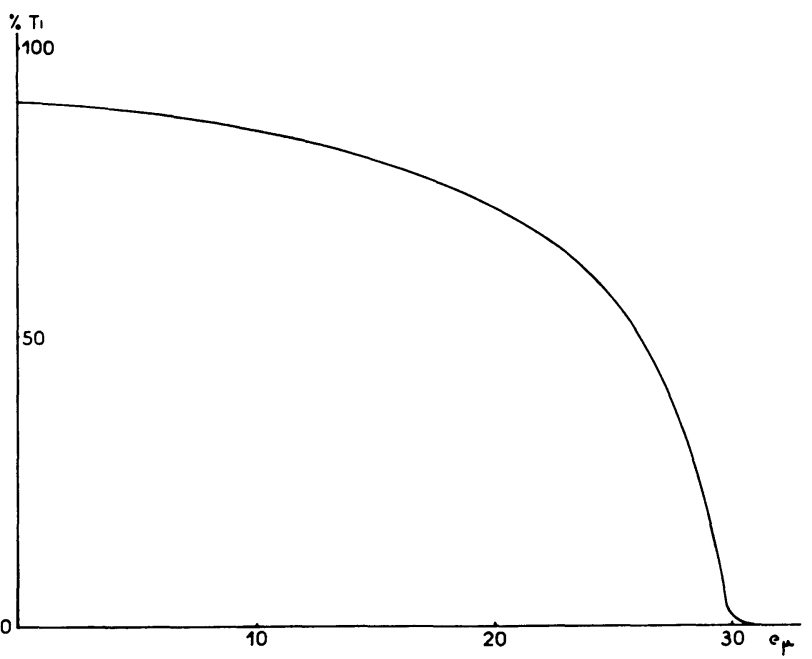

FIG. 1. - Composition d'une zone superficielle de solutions solides $\mathrm{Nb}$-Ti réalisée à $1000^{\circ} \mathrm{C}$ en $2 \mathrm{~h} \mathrm{45^{ \prime }}$.

Toutefois, contrairement à ce que ces résultats pourraient laisser supposer, la zone de diffusion n'est pas parfaitement uniforme. Elle présente des irrégularités dans toutes les parties de l'échantillon qui sont en contact avec les copeaux ; la solution solide y est en effet plus riche en titane, sa teneur peut approcher de $100 \%$, et la pénétration est plus grande.

Il apparaît donc que la géométrie du système de 
cémentation est également un facteur important, le transfert des réactifs gazeux étant influencé par la distance source-substrat.

Les irrégularités du revêtement sont encore plus marquées si les copeaux de titane sont dilués dans une poudre inerte. Elles disparaissent par contre lorsque le donneur est constitué d'un alliage pulvérulent de titane et d'un métal non réactif tel que le molybdène ou le niobium lui-même. Dans ces conditions, la zone de diffusion est uniforme mais sa composition dépend non seulement de la température et de la durée du traitement mais aussi de la composition de l'alliage et de la pression d'iode.

On remarque enfin que l'influence de la température sur la croissance du revêtement ne se traduit pas par une simple loi exponentielle d'Arrhénius, ce qui confirme l'interférence entre la réaction chimique de transport et le processus de diffusion à l'état solide.

Ainsi l'application du procédé est assez délicate et nécessite chaque fois une mise au point particulière.

Siliciuration des zones superficielles de solutions solides niobium-titane. - Des difficultés ont été également rencontrées dans la siliciuration des zones superficielles de solutions solides niobium-titane. Lorsque celles-ci sont très riches en titane (70 à $80 \%$ en masse), les revêtements de siliciures sont peu adhérents et ont tendance à se désagréger au refroidissement.

Cette fragilité peut provenir du fait que ces revêtements sont biphasés puisque le disiliciure de niobium ne dissout que 45 moles pour cent environ de disiliciure de titane, au-dessus de $1000^{\circ} \mathrm{C}$ [6].

III. Revêtements modifiés par le chrome. - Le niobium pur a été également soumis à des essais systématiques de cémentation par des alliages chromesilicium de différentes compositions. Certains d'entre eux ont permis de réaliser des revêtements uniformes comportant une couche superficielle riche en chrome et ceux-ci méritaient donc un examen plus approfondi.

Des résultats analogues ont d'ailleurs été obtenus en traitant par les mêmes alliages des échantillons préalablement siliciurés.

Les données de J. M. Goldschmidt [7] sur le système ternaire niobium-chrome-silicium ont facilité l'étude des revêtements et il a paru utile de reproduire sur la figure 2, le diagramme d'équilibre à $1000^{\circ} \mathrm{C}$ établi par cet auteur ; les deux métaux forment avec le silicium plusieurs phases ternaires mais la miscibilité de leurs disiliciures est très faible même à plus haute température.

1. TRAITEMENT DU NiOBIUM PAR DES ALliages CHROME-SILICIUM. - Influence de la composition de l'alliage donneur. - Des essais de cémentation activée par l'iode ont été effectués avec des alliages de différentes compositions dans l'intervalle $\mathrm{Cr}_{5} \mathrm{Si}_{3}-\mathrm{Si}$ du système binaire.

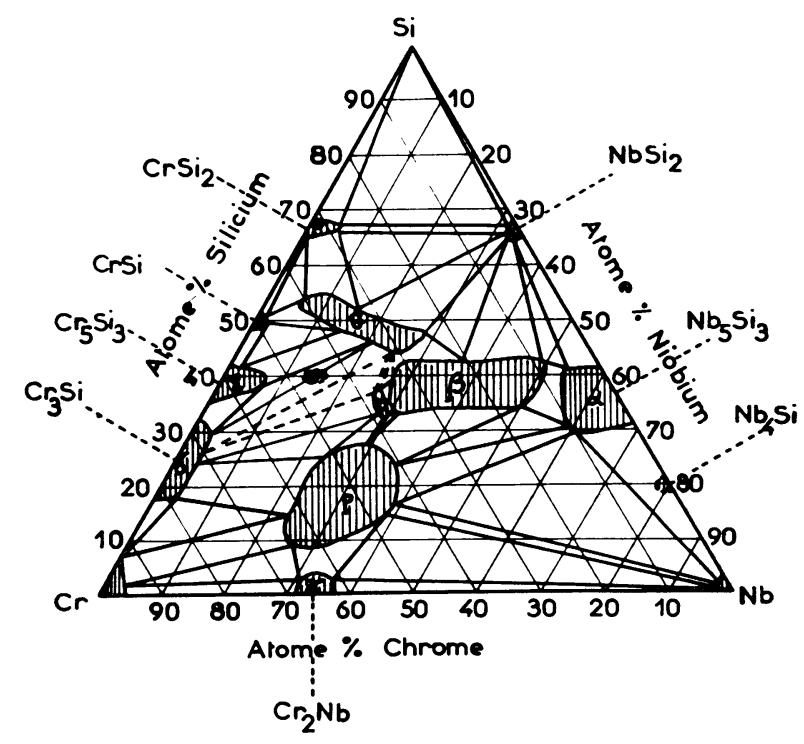

FIG. 2. - Diagramme d'équilibre à $1000^{\circ} \mathrm{C}$ du système $\mathrm{Nb}$ $\mathrm{Cr}-\mathrm{Si}$ (d'après [7])

Pour cette étude, la température a été fixée à $1150^{\circ} \mathrm{C}$ et la pression d'iode à 1 atmosphère.

Les résultats obtenus suivant la composition du donneur se résument de la façon suivante :

\begin{tabular}{|c|c|}
\hline $\begin{array}{l}\text { Composition du } \\
\text { donneur } \mathrm{Cr}_{1-x} \mathrm{Si}_{x} \\
\text { (en atomes) }\end{array}$ & Résultats \\
\hline- & - \\
\hline $\begin{array}{l}x=0,37\left(\mathrm{Cr}_{5} \mathrm{Si}_{3}\right) \\
0,40 \leqslant x \leqslant 0,45\end{array}$ & $\begin{array}{l}\text { attaque du niobium } \\
\text { revêtements irréguliers, à } 5 \text { couches et } \\
\text { contenant du chrome }\end{array}$ \\
\hline $0,50 \leqslant x \leqslant 0,65$ & $\begin{array}{l}\text { revêtements uniformes, à } 4 \text { couches et } \\
\text { contenant du chrome }\end{array}$ \\
\hline$x \geqslant 0,67\left(\mathrm{CrSi}_{2}\right)$ & $\begin{array}{l}\text { revêtements de siliciures de niobium pra- } \\
\text { tiquement exempts de chrome }\end{array}$ \\
\hline
\end{tabular}

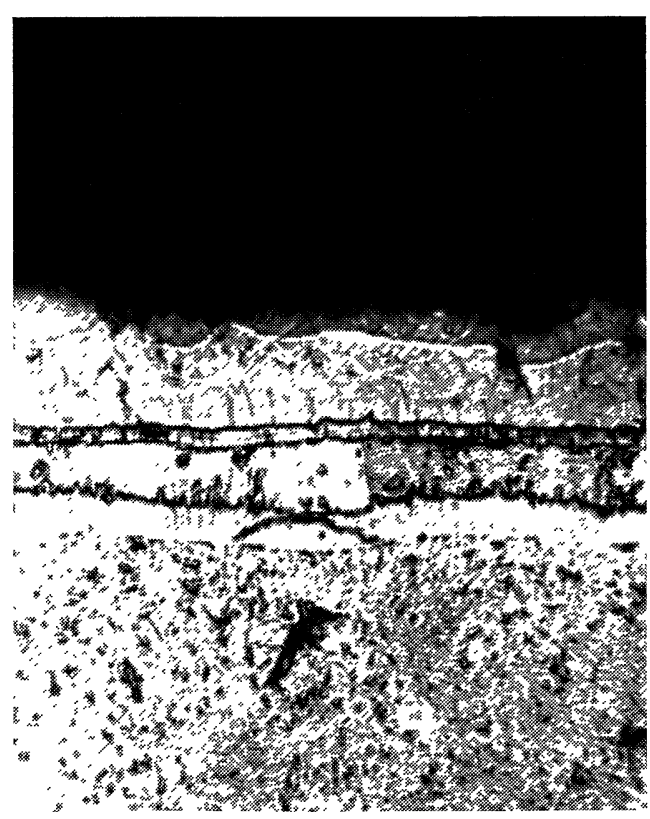

Fig. 3. - Revêtement résultant d'un traitement de $24 \mathrm{~h}$ à $1150{ }^{\circ} \mathrm{C}$ par l'alliage $\mathrm{Cr}_{0,5}-\mathrm{Si}_{0,45}(1000 \times)$. 
Ce sont naturellement les deux types de revêtement contenant du chrome qui ont plus particulièrement retenu notre attention.

Revêtements formés à partir de $\mathrm{Cr}_{1-x} \mathrm{Si}_{x}$ avec $0,40 \leqslant x \leqslant 0,45$. - Ces revêtements sont très irréguliers (Fig. 3). Ils comportent toujours cinq couches successives dont les compositions ont été déterminées par diffraction $X$ et microanalyse à la sonde électronique (Fig. 4). Ce sont les suivantes :

$-\mathrm{Nb}_{5} \mathrm{Si}_{3}$,

- $\mathrm{NbSi}_{2}$ exempt de chrome,

$-\beta$, phase ternaire de composition :

$\mathrm{Cr} \simeq 21$ at. $\% ; \mathrm{Nb} \simeq 37$ at. $\% ; \mathrm{Si} \simeq 42$ at. $\%$, et de structure quadratique du type D $8 \mathrm{~m}\left(\mathrm{~W}_{5} \mathrm{Si}_{3}\right)$,

$-\theta$, phase ternaire de composition $\left(\mathrm{Cr}_{1-x} \mathrm{Nb}_{x}\right)_{6} \mathrm{Si}_{5}$ avec $x$ variant entre 0,2 et 0,4 environ. Cette phase est isomorphe avec le siliciure $\mathrm{V}_{6} \mathrm{Si}_{5}$, du groupe de symétrie Ibam [8],

$-\eta$ solution solide de niobium dans $\mathrm{Cr}_{5} \mathrm{Si}_{3}$ à très faible teneur en niobium.

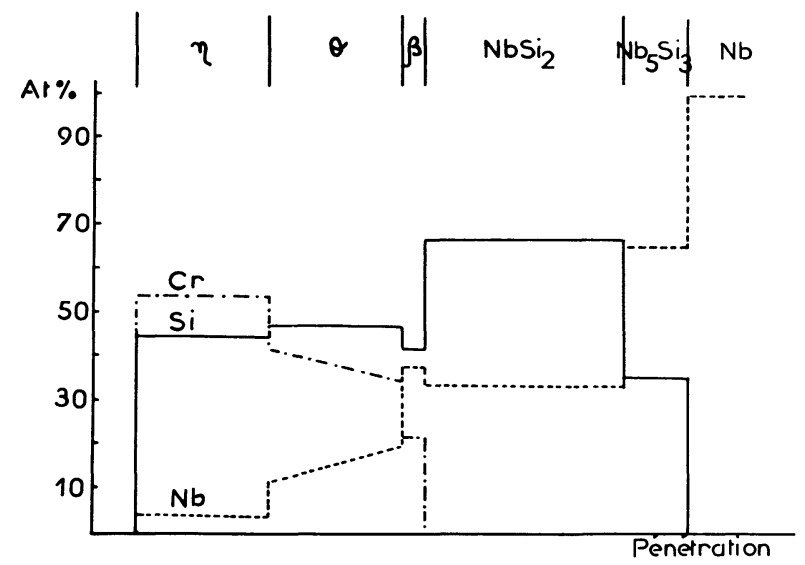

Fig. 4. - Composition des revêtements résultant de traitements à $1150^{\circ} \mathrm{C}$, par des alliages $\mathrm{Cr}_{1-x} \mathrm{Si}_{x}$ avec $0,40 \leqslant x \leqslant 0,45$.

La couche superficielle de cette phase $\eta$ est très peu uniforme et c'est surtout elle qui est à l'origine des irrégularités $\mathrm{du}$ revêtement.

Revêtements formés à partir de $\mathrm{Cr}_{1-x} \mathrm{Si}_{x}$ avec $0,50 \leqslant x \leqslant 0,65$. - Des revêtements uniformes sont par contre obtenus en traitant le niobium par le monosiliciure de chrome ou des mélanges de mono-et disiliciures.

Leur examen micrographique montre qu'ils sont tous constitués de quatre couches (Fig. 5) qui sont identiques, d'après leur microanalyse (Fig. 6), aux quatre couches internes des revêtements précédents ; seule la phase $\eta$ est absente.

Les deux constituants essentiels sont la phase $\theta$ et le disiliciure de niobium, et il est clair que la couche de ce siliciure devient relativement plus épaisse lorsque le donneur est enrichi en silicium. Cet enrichissement se traduit également par une diminution de l'épaisseur des couches de $\mathrm{Nb}_{5} \mathrm{Si}_{3}$ et de la phase $\beta$ qui restent toujours très minces par rapport aux deux autres. a)

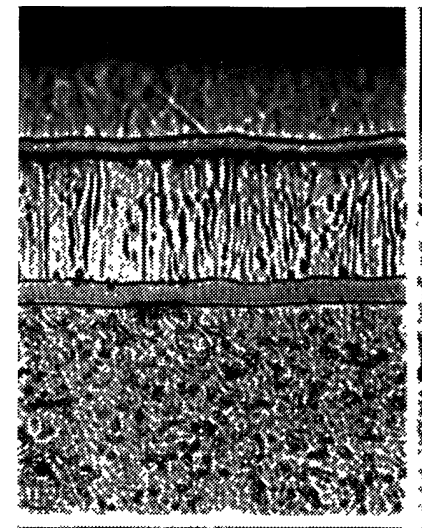

b)

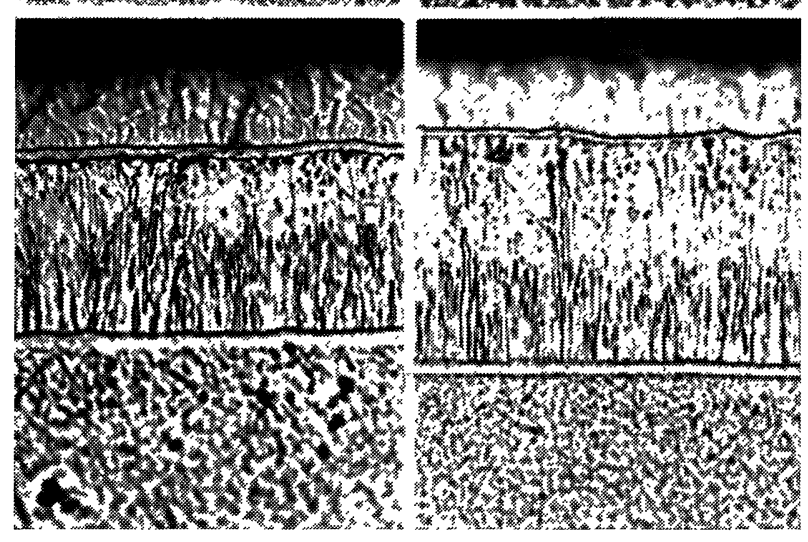

c)

d)

Fig. 5. - Revêtements résultant d'un traitement de $24 \mathrm{~h}$ à $1150^{\circ} \mathrm{C}$ par des alliages $\mathrm{Cr}_{1-x} \mathrm{Si}_{x}$ avec $0,50 \leqslant x \leqslant 0,65(1000 \times)$

a) $\mathrm{Cr}_{0,50} \mathrm{Si}_{0,50}$

b) $\mathrm{Cr}_{0,45} \mathrm{Si}_{0,55}$

c) $\mathrm{Cr}_{0,40} \mathrm{Si}_{0,60}$

d) $\mathrm{Cr}_{0,35} \mathrm{Si}_{0,65}$.

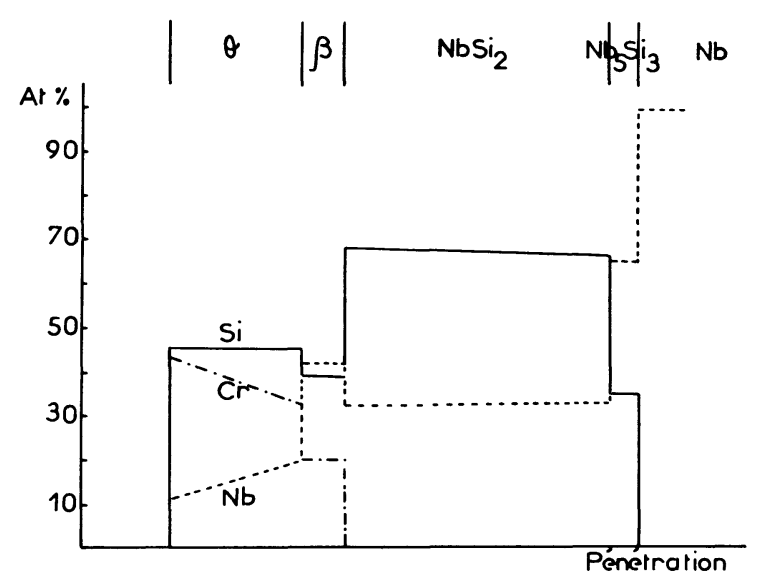

FIG. 6. - Composition des revêtements résultant de traitements à $1150^{\circ} \mathrm{C}$, par des alliages $\mathrm{Cr}_{1 x-} \mathrm{Si} x$ avec $0,50 \leqslant x \leqslant 0,65$.

2. ETUDE DES REVÊTEMENTS FORMÉS A PARTIR DE L'Alliage $\mathrm{Cr}_{0,4} \mathrm{Si}_{0,6}$. - Les revêtements à couche superficielle de la phase $\theta$, et plus particulièrement ceux obtenus à partir de l'alliage $\mathrm{Cr}_{0,4} \mathrm{Si}_{0,6}$, ont été sélectionnés en raison de leur régularité et de la bonne reproductibilité de leur fabrication.

Leur cinétique de croissance a été examinée et ils 
ont été soumis à quelques essais d'oxydation et de siliciuration.

Cinétique de formation. - Il a d'abord été vérifié que la pression de l'agent activant n'est pas un paramètre important de la cémentation; dans une série d'essais isochrones à $1150^{\circ} \mathrm{C}$, la pression d'iode a été modifiée entre 0,1 et 1 atmosphère sans conséquence pour la composition du revêtement.

La cinétique a été ensuite examinée à 1150 et $1250^{\circ} \mathrm{C}$; les variations de l'épaisseur des couches sont représentées sur les figures 7 et 8 en fonction de la racine carrée du temps.

Les isothermes sont sensiblement linéaires mais présentent toutes une ordonnée à l'origine positive ; il semble donc que la diffusion dans l'état solide ne soit pas immédiatement le processus régulateur.

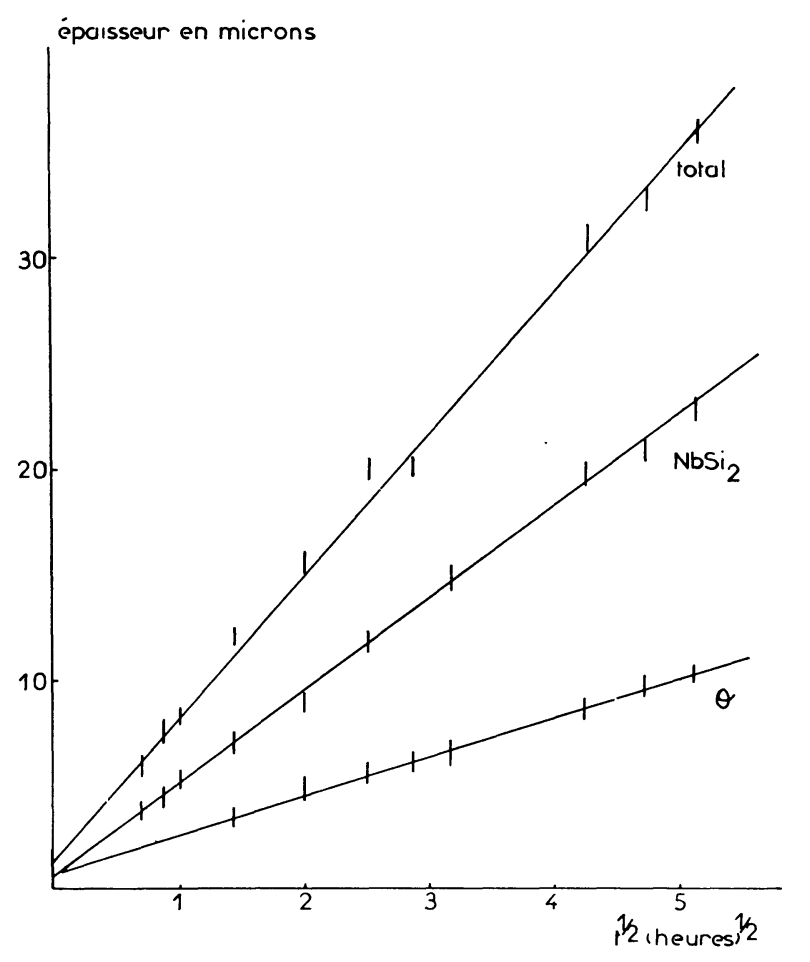

FIG. 7. - Croissance du revêtement au cours du traitement par l'alliage $\mathrm{Cr}_{0,4} \mathrm{Si}_{0,6}$, à $1150^{\circ} \mathrm{C}$.

La cémentation s'accélère nettement lorsque la température de traitement augmente; les pentes des isothermes correspondant au disiliciure et à la phase $\theta$ sont à peu près doublées entre 1150 et $1250^{\circ} \mathrm{C}$; leur rapport varie aussi avec la température mais assez faiblement.

\begin{tabular}{cccc}
$\substack{\text { Température } \\
\left({ }^{\circ} \mathrm{C}\right)}$ & \multicolumn{2}{c}{ Constantes paraboliques } & $\frac{k \mathrm{NbSi}_{2}}{k \theta}$ \\
& $k \theta$ & $k \mathrm{NbSi}_{2}$ & \\
- & - & - & - \\
1250 & 3,5 & 10 & $\sim 2,9$ \\
1150 & 1,9 & 4,5 & $\sim 2,4$ \\
$\frac{k 1250}{k 1150}$ & $\sim 1,8$ & $\sim 2,2$ &
\end{tabular}

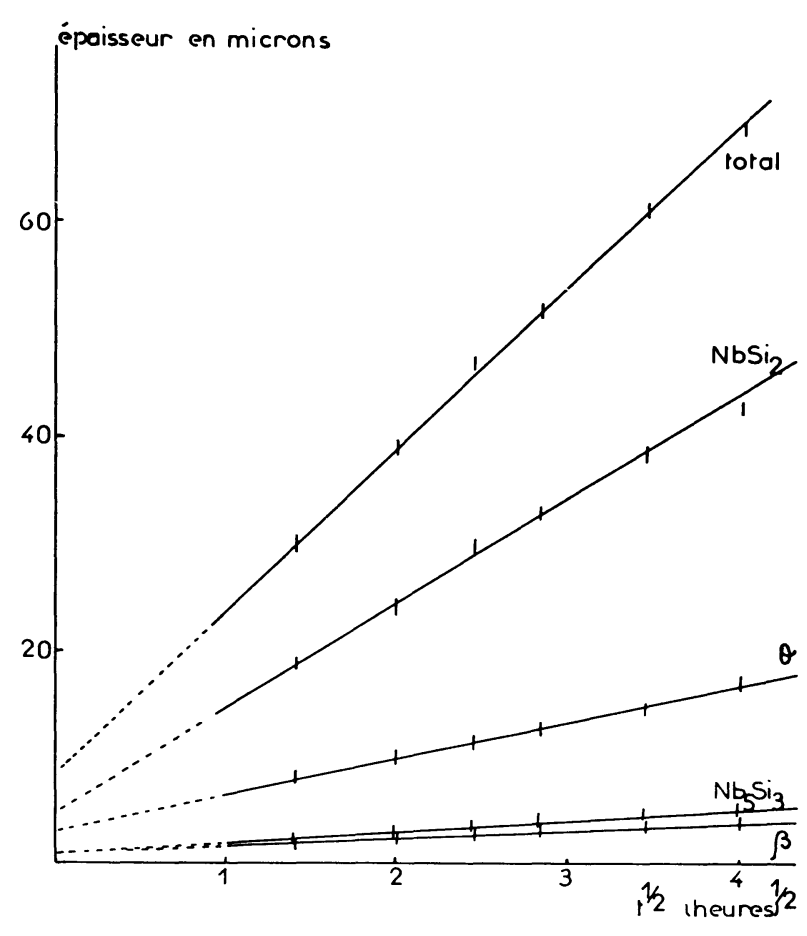

FIG. 8. - Croissance du revêtement au cours du traitement par l'alliage $\mathrm{Cr}_{0,4} \mathrm{Si}_{0,6}$, à $1250^{\circ} \mathrm{C}$.

Compte tenu des écarts à l'origine, le rapport $\frac{e \mathrm{NbSi}_{2}}{e \theta}$ des épaisseurs réelles varie entre 2,1 et 2,3 à $1150^{\circ} \mathrm{C}$ et entre 2,5 et 2,6 à $1250^{\circ} \mathrm{C}$.

Résistance à l'oxydation. - Une série d'échantillons a été soumise à des essais d'oxydation; il s'agissait de plaquettes de $20 \times 20 \times 1 \mathrm{~mm}$ dont le revêtement avait été réalisé par un traitement de $24 \mathrm{~h}$ à $1150{ }^{\circ} \mathrm{C}$ avec l'alliage $\mathrm{Cr}_{0,6} \mathrm{Si}_{0,6}$, et avait donc la constitution suivante :

- couche superficielle de la phase $\theta$ de 10 microns, - couche intermédiaire de disiliciure de niobium de 22 à 23 microns.

On pouvait craindre que la résistance à l'oxydation de ces revêtements soit limitée à haute température car leur couche superficielle est assez pauvre en silicium. Des essais isothermes ont tout de même été effectués à l'air à $1150^{\circ} \mathrm{C}$; ils ont permis de situer la durée de vie des pièces protégées entre 200 et $250 \mathrm{~h}$.

D'autre part, nous nous sommes plus particulièrement intéressés au comportement de ce système de protection dans le domaine des températures moyennes comprises entre 600 et $950^{\circ} \mathrm{C}$; on sait en effet que dans ces conditions, plusieurs disiliciures dont celui de niobium sont très sensibles à l'oxydation.

Des essais de $100 \mathrm{~h}$ ont donc été réalisés en thermobalance, sous une atmosphère d'oxygène pur et sec, aux quatre températures : $650,750,850$ et $930^{\circ} \mathrm{C}$. Le poids des échantillons n'a pratiquement pas varié au cours de ces expériences et la seule modification observée concerne la couleur des revêtements qui 
évolue très rapidement du gris métallique initial au brun à 650 et $750^{\circ} \mathrm{C}$ et au vert à plus haute température.

Les échantillons testés à 750 et $850^{\circ} \mathrm{C}$ ont d'ailleurs été soumis ultérieurement à des traitements de $700 \mathrm{~h}$ aux mêmes températures mais à l'air ambiant; ils n'ont subi aucun dommage important au cours de cette nouvelle oxydation.

Ce système de protection semble donc nettement plus efficace aux températures inférieures à $1000^{\circ} \mathrm{C}$ que les revêtements de siliciures ou aluminiures de niobium. Cette meilleure résistance pourrait être liée au fait que l'oxydation ne conduit plus à la formation d'oxydes de niobium pur ; après des traitements de longue durée à des températures comprises entre 750 et $1100^{\circ} \mathrm{C}$, l'examen du revêtement par diffraction $\mathrm{X}$ fait seulement apparaître le spectre caractéristique de l'oxyde de chrome $\mathrm{Cr}_{2} \mathrm{O}_{3}$ et quelques raies supplémentaires que l'on peut attribuer à la phase ternaire $\mathrm{CrNbO}_{4}$.

Il convient toutefois de remarquer que la protection est très mauvaise aux angles et que ceux-ci constituent généralement l'amorce d'une oxydation très rapide du métal de base. Les revêtements à base de chrome présentent en outre l'inconvénient d'être fragiles; ce défaut se manifeste en particulier lors de la préparation de coupes métallographiques où le polissage provoque souvent la fissuration et même le décollement de la couche superficielle.

Siliciuration. - Des échantillons préalablement traités par l'alliage $\mathrm{Cr}_{0,4} \mathrm{Si}_{0,6}$ ont été soumis à des essais de siliciuration.

Une siliciuration à $1000^{\circ} \mathrm{C}$, activée par le tétrachlorure de silicium, fournit des revêtements de disiliciures comportant une couche superficielle riche en chrome (Fig. 9 et 10).

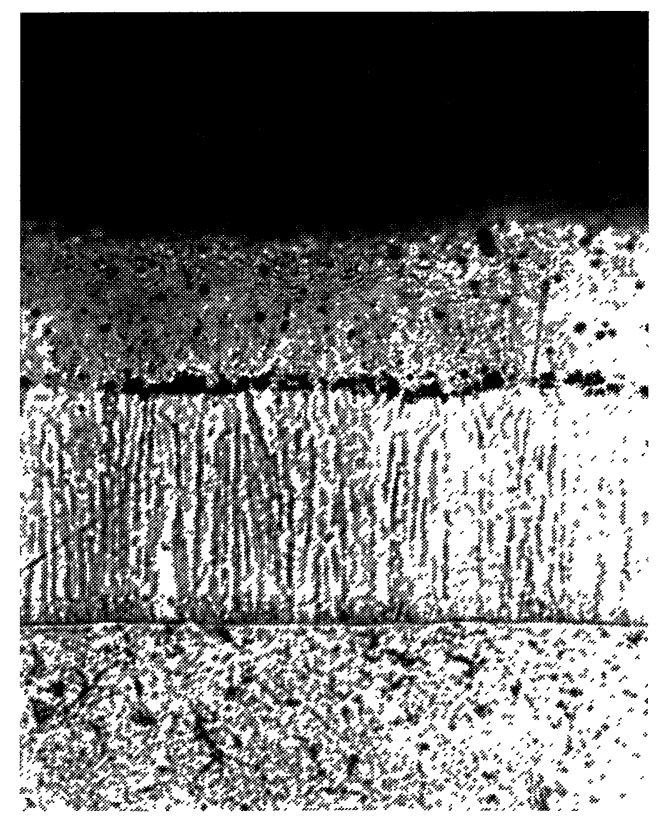

FIG. 9. - Echantillon traité par l'alliage $\mathrm{Cr}_{0,4} \mathrm{Si}_{0,6}$ à $1150^{\circ} \mathrm{C}$ pendant $24 \mathrm{~h}$ puis par le silicium à $1000^{\circ} \mathrm{C}$ pendant $4 \mathrm{~h}(1000 \times)$.
Cette transformation est assez délicate ; le décollement de cette couche a été observé dans plusieurs essais effectués avec l'iode comme agent activant; il semble tout de même que son adhérence soit meilleure lorsque la durée de traitement est suffisamment longue non seulement pour transformer les phases $\theta$ et $\beta$ en disiliciures mais aussi pour assurer une siliciuration supplémentaire du métal de base.

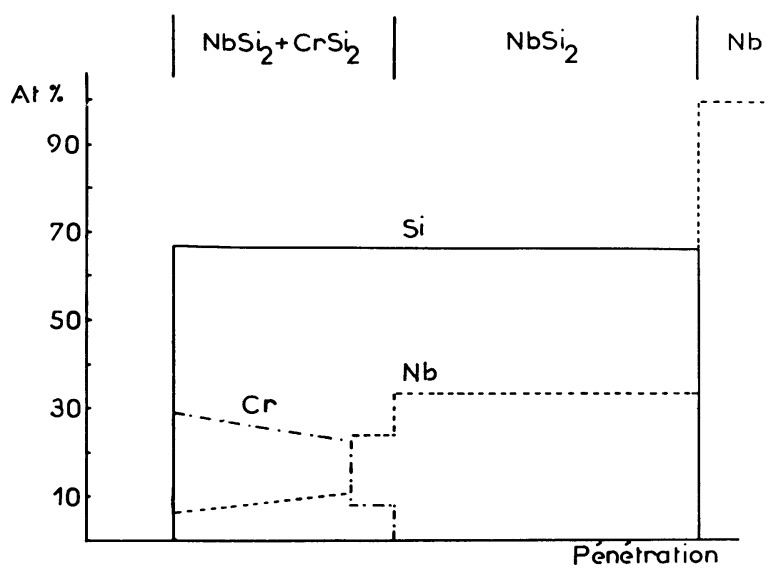

Fig. 10. - Composition des revêtements résultant d'un double traitement, par l'alliage $\mathrm{Cr}_{0,4} \mathrm{Si}_{0,6}$ puis par le silicium pur.

3. TRAITEMENT DU NIOBIUM PRÉALABLEMENT SILICIURÉ PAR DES ALLIAGES $\mathrm{Cr}_{1-x} \mathrm{Si}_{x}$. - Il est également possible de réaliser des revêtements associant le chrome au silicium en soumettant le niobium à deux traitements successifs;

- une siliciuration qui fournit un revêtement de disiliciure.

- un traitement par un alliage chrome-silicium choisi dans le domaine $\mathrm{CrSi}_{-} \mathrm{CrSi}_{2}$, avec l'iode comme agent activant.

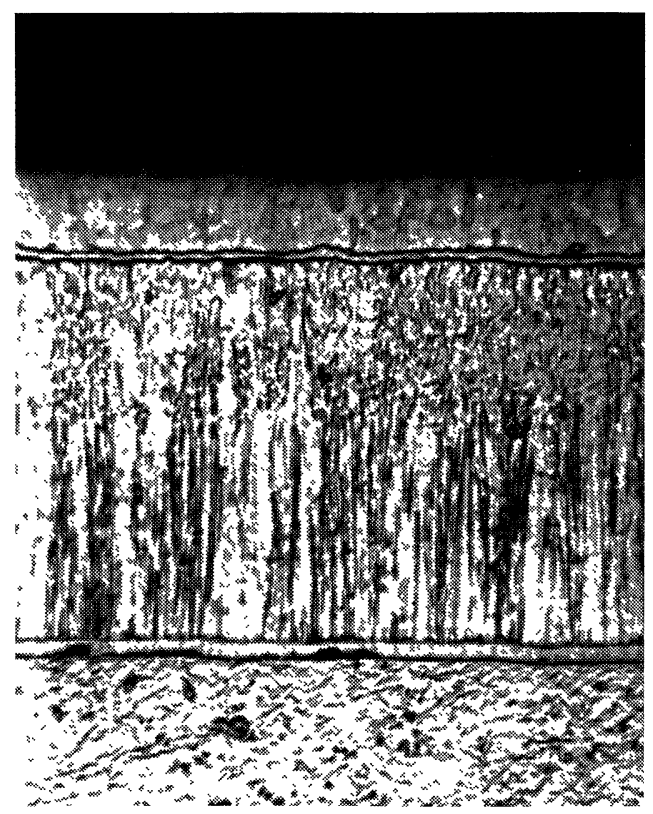

Fig. 11. - Echantillon préalablement recouvert d'une couche de disiliciure de $28 \mu$, puis traité par l'alliage $\mathrm{Cr}_{0,4} \mathrm{Si}_{0,6}$ à $1150^{\circ} \mathrm{C}$ pendant $24 \mathrm{~h}(1000 \times)$. 
Ce dernier a le même effet que s'il était réalisé directement sur le métal de base ; il provoque la formation de deux couches superficielles constituées respectivement des phases ternaires $\theta$ et $\beta$, la couche de disiliciure de niobium se développe et une fine sous couche de $\mathrm{Nb}_{5} \mathrm{Si}_{3}$ apparaît (Fig. 11).

La cinétique de formation de ces revêtements est très voisine de celle qui a été observée dans les traitements directs.

Elle est également très sensible à la température ; les lois de croissance isotherme de la couche superficielle sont à peu près identiques dans les deux cas ; la croissance de la couche de disiliciure n'est que faiblement ralentie.

Ces analogies apparaissent clairement dans le tableau suivant qui précise la constitution des revête-

\begin{tabular}{cccccc} 
Epaisseur $(\mu)$ & \multicolumn{5}{c}{ Epaisseur des couches du revêtement $(\mu)$} \\
initiale & $\mathrm{NbSi}_{2}$ & $\theta$ & $\beta$ & $\mathrm{Nb}_{5} \mathrm{Si}_{3}$ \\
$\begin{array}{c}\mathrm{de} \mathrm{NbSi}{ }_{2} \\
e_{0}\end{array}$ & $e_{1}$ & $e_{1}-e_{0}$ & $e_{2}$ & $e_{3}$ & $e_{4}$ \\
- & - & - & - & - & - \\
0 & 23 & 23 & 10 & 2 & $1-2$ \\
12 & 35 & 23 & 10 & 2 & $1-2$ \\
21 & 42 & 21 & 10 & 2 & $1-2$ \\
28 & 48 & 20 & 10 & 2 & $1-2$ \\
35 & 51 & 16 & 10 & 2 & $1-2$
\end{tabular}

ments formés sur des échantillons de niobium purs et préalablement siliciurés, par un traitement de $24 \mathrm{~h}$ à $1150^{\circ} \mathrm{C}$ avec l'alliage $\mathrm{Cr}_{0,4} \mathrm{Si}_{0,6}$.

Conclusion. - L'étude de la cémentation du niobium par les siliciures de titane et de chrome a été entreprise.

Ce procédé ne permet pas de réaliser directement des revêtements associant le titane et le silicium et il faut donc déposer successivement ces deux éléments. Cette dernière méthode présente quelques difficultés dans :

- la réalisation de zones superficielles de solutions solides riches en titane et parfaitement uniformes,

- la transformation de ces solutions solides en disiliciures de niobium et de titane adhérents au substrat.

Par contre, les cémentations par certains alliages chrome-silicium fournissent des revêtements de siliciures riches en chrome. La croissance et quelques propriétés de ceux-ci ont été examinées.

Il est envisagé d'appliquer un traitement analogue à des échantillons de niobium préalablement titanisés, en vue de réaliser des revêtements associant les trois éléments : titane, chrome et silicium.

L'étude sera également étendue aux cémentations par des siliciures de fer et des alliages ternaires chromefer-silicium.

\section{Bibliographie}

[1] GAdD (J.-D.), JeFFERYS (R. A.), « Design data study for coated columbium alloys ), Final Summary Report ER, Bureau of Naval Weapons RRMA, Contract no 62-0098-C, 1963.

[2] Stetson (A. R.), Crociani (D.) et Brentnall (W.), ("The V-(Cr-Ti)-Si coating for columbium - base alloys ), 11th Meeting of the Refractory Composite Working Group, Los Angeles, Calif., 25-27 jan. 1966.

[3] Priceman (S.), Sama (L.), SAE Journal, 1967, 75, 46.

[4] Stecher (P.), Lux (B.) et FunK (R.), Entropie, 1968, 39.
[5] Albrecht (J. M.), Ouvrard (J.), Wandji (R.) et RoQues (B.), C. R. Acad. Sci., 1969, 268 C, 1518.

[6] GladyshevskiI (E. I.), LaKh (V. I.), Skolozdra (R. V.) et Stadnik (B. I.), Poroshkov. Metallurg., 1964, 22, 15.

[7] Goldschmidt (M. J.), Brand (J. A.), J. less-common Metals, 1961, 3, 34.

[8] Herpin (P.), Spinat (P.), Hallais (J.), Fruchart (R.), Albrecht (J. M.), Ouvrard (J.), C. R. Acad. Sci., 1969, 268 C, 1750. 